\title{
érythème polymorphe : à propos de 3 cas cliniques
}

RÉSUMÉ L'érythème polymorphe (EP) est un syndrome éruptif aigu. Il peut survenir avec ou sans atteinte muqueuse. Sa lésion élémentaire au niveau muqueux est une bulle qui rapidement éclate et laisse des érosions plus ou moins superficielles et douloureuses. Au niveau cutané, la lésion en cocarde siège essentiellement au niveau des extrémités. Le diagnostic positif est clinique et anatomopathologique. Le traitement de l'EP comporte trois volets, l'un symptomatique, l'autre étiologique, et enfin un traitement de fond qui fait appel aux corticoïdes, mais aussi au levamisole, dapsone, thalidomide, etc. L'évolution, bien que généralement bénigne, peut être émaillée de périodes de récidives.

Ihsane BENYAHYA

Professeur de l'enseignement supérieur et chef de service d'odontologie chirurgicale,

rue Abou Alaa Zahar,

Casablanca, Maroc.

\section{KISSI}

Titulaire du diplôme national de spécialité en odontologie chirurgicale, 42, bd Moulay Driss 1er, apprt 9,

3e étage,

Casablanca, Maroc.

\section{K. GUESSOUS}

Docteur en médecine dentaire,

7, rue la place, quartier des hôpitaux, résidence Yacout $B$,

Casablanca, Maroc.
Le rôle du chirurgien-dentiste étayé à travers trois observations est fondamental dans le diagnostic et la prise en charge thérapeutique en collaboration avec le dermatologue et l'ophtalmologue.

\section{MOTS CLÉS}

érythème polymorphe

syndrome de Stevens Johnson

stomatite bulleuse

cocarde 


\section{introduction}

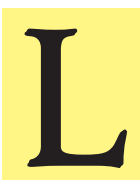

'érythème polymorphe (EP) est une dermatose dont la dénomination témoigne $\mathrm{du}$ polymorphisme des lésions observées chez un même patient. Cliniquement, elle se manifeste par des lésions tantôt maculeuses, tantôt papuleuses et bulleuses avec des éléments en cible caractéristiques appelés cocardes.

\section{cas clinique $\mathbf{n}^{\circ} 1$ (fig. 1 à 7 )}

Madame B., âgée de 28 ans, s'est présentée en 2003 au service d'odontologie chirurgicale du centre de consultations et de traitements dentaires (CCTD) pour des douleurs au niveau de toute la cavité buccale.

L'interrogatoire a révélé que l'état de santé général de la patiente était bon sans antécédents médico-chirurgicaux particuliers.

L'histoire de la maladie débute en 2002, date à laquelle Madame B. était hospitalisée pour une dermatose bulleuse dont on n'a pas précisé le diagnostic et qui a bien répondu au traitement par un antiinflammatoire stéroïdien.

Plus tard, au cours de la même année, il y a eu une récidive. Depuis, la patiente rapporte des phases de rémission et d'exacerbation de la maladie.

L'examen clinique endobuccal a révélé des lésions buccales, localisées au niveau de la face interne de la joue (fig. 1), et le bord de la langue (fig. 2). Ces lésions étaient érosives par endroits et végétantes en d'autres. Sur le plan
Les formes cliniques sont diverses et variées avec ou sans atteinte muqueuse. L'étiologie de l'érythème polymorphe est obscure. Le traitement est symptomatique. À travers 3 cas cliniques traités et suivis au service d'odontologie chirurgicale de Casablanca, cet article se propose de traiter les aspects cliniques et thérapeutiques de l'érythème polymorphe. cutané, elle présentait des lésions punctiformes érythémateuses et prurigineuses localisées au niveau des mains, de la cheville, de la nuque et de l'avantbras (fig. 3, 4, 5).

Ces lésions en cocarde simulaient l'aspect d'un EP. Une biopsie est réalisée au niveau de la face interne des joues et l'examen anatomopathologique a confirmé la présence d'un EP (fig. 6).

À ce stade, le diagnostic d'un EP majeur a été posé. Le traitement instauré était à base d'anti-inflammatoires stéroïdiens : prednisolone (Solupred $\left.{ }^{\circledR}\right) 20 \mathrm{mg}$, à raison de $4 \mathrm{cp} / \mathrm{j}$ pendant 5 jours puis en dose régressive $(-20 \mathrm{mg}$ tous les 5 jours).

Au niveau local, une motivation à l'hygiène bucco-dentaire et des soins dentaires lui ont été prodigués.

Cinq jours plus tard, il y a eu une bonne évolution (fig. 7) mais sept mois plus tard, une autre rechute a été constatée, et la patiente a été adressée au service de dermatologie pour une prise en charge. 

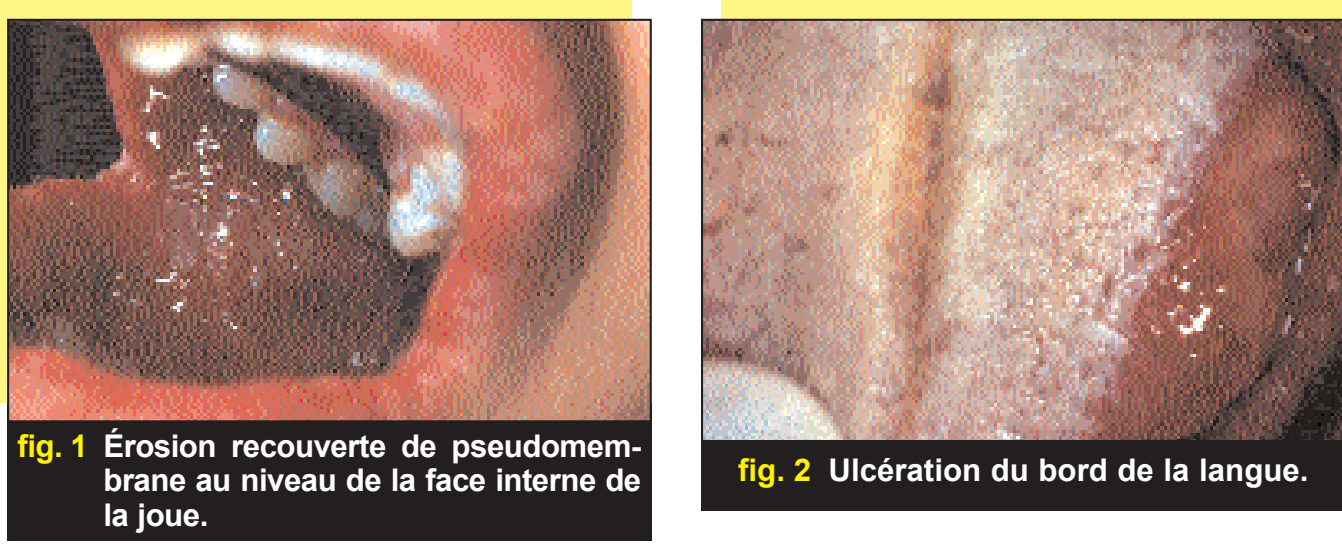

fig. 2 Ulcération du bord de la langue.
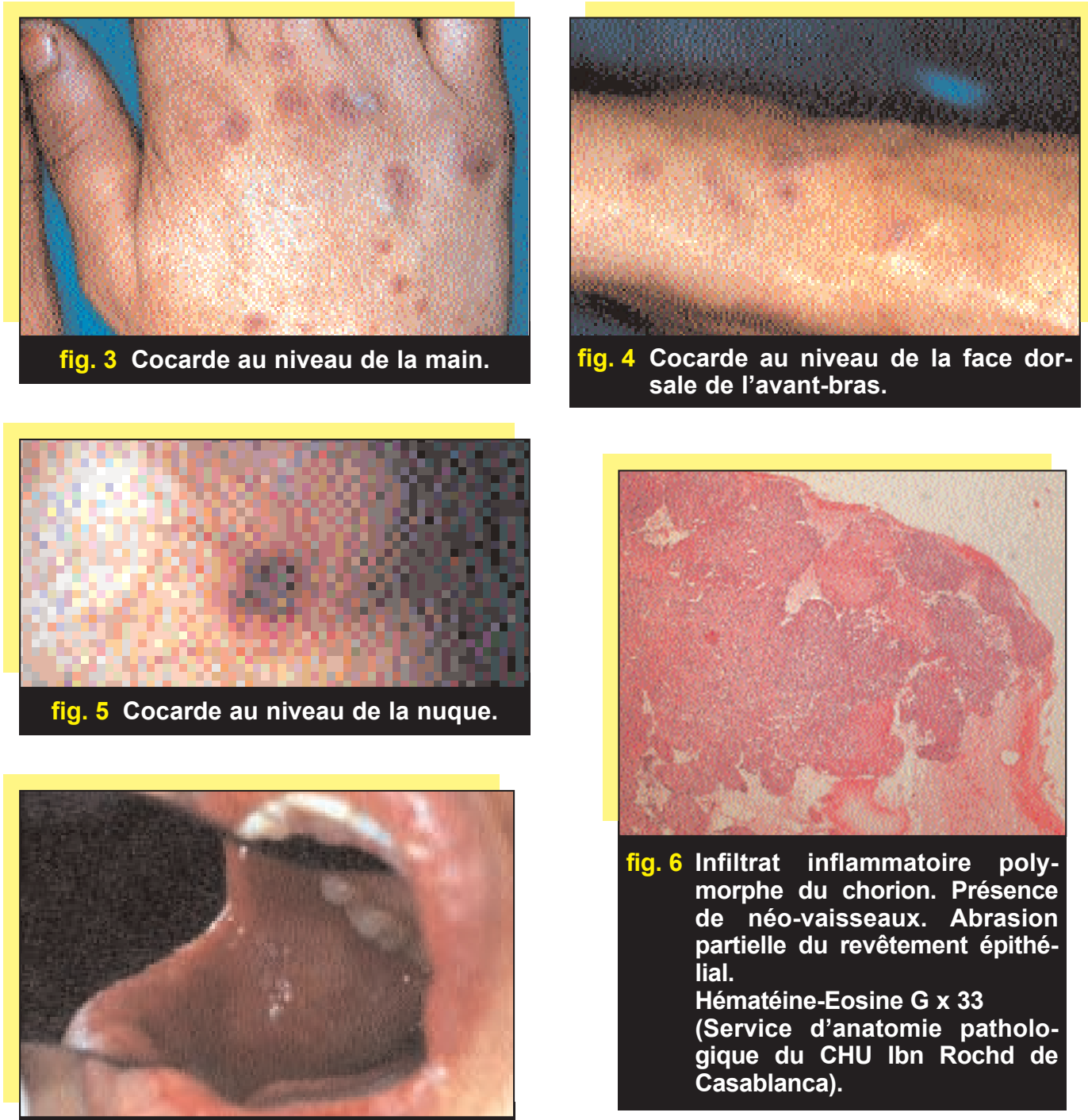

fig. 7 Face interne de la joue après quelques jours de traitement. 
La fréquence des récidives ainsi qu'une poussée herpétique avant l'éruption bulleuse a confirmé la nature et l'étiologie herpétique de l'érythème polymorphe chez cette patiente.

Cette dernière a été mise sous aciclovir à raison de $400 \mathrm{mg}, 2$ fois/jour pendant 6 mois. Pendant les deux premières années, à l'arrêt du traitement, la patiente présentait des récidives. Actuellement, aucune récidive n'a été notée en 2005.

\section{cas clinique $n^{\circ} 2$ (fig. 8 à 12)}

Monsieur H., âgé de 35 ans, s'est adressé au service d'odontologie chirurgicale du CCTD pour des douleurs buccales à l'origine d'une gêne fonctionnelle très importante entravant l'alimentation.

Les antécédents médico-chirurgicaux du patient ont révélé une maladie de Behçet confirmée et traitée par colchicine à raison de $1 \mathrm{cp} / \mathrm{j}$.

L'examen clinique a révélé la présence de lésions bulleuses érosives au niveau de la pointe de la langue et croûteuses sur la lèvre supérieure et inférieure (fig. 8, 9, 10). Sur le plan cutané, aucune lésion n'a été relevée.

Devant ces signes cliniques, le diagnostic d'érythème polymorphe a été supposé et confirmé par un examen anatomopathologique. Ce dernier a révélé la pré-

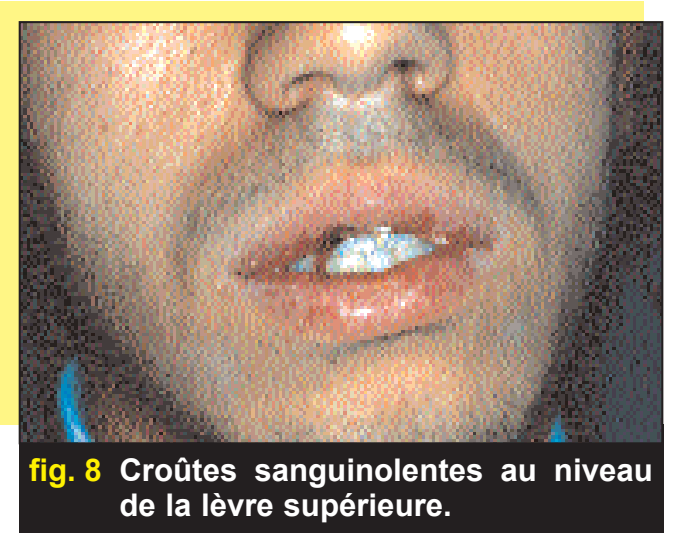

sence d'une bulle intra-épithéliale à clivage basal non acantholytique dont le toit renferme des kératinocytes nécrosés. Le patient présentait donc un EP buccal qui n'avait a priori aucun rapport avec la maladie de Behçet.

Le traitement instauré chez ce patient a été à base d'anti-inflammatoire stéroïdien, en l'occurrence prednisolone (Solupred ${ }^{\circledR}$ ) $20 \mathrm{mg}, 4 \mathrm{cp} / \mathrm{j}$ associé à une motivation à l'hygiène bucco-dentaire et des soins dentaires.

Le protocole était le suivant : $80 \mathrm{mg} / \mathrm{j}$ pendant 4 jours, puis $60 \mathrm{mg} / \mathrm{j}$ pendant 4 jours, puis $40 \mathrm{mg} / \mathrm{j}$ pendant 4 jours, et $20 \mathrm{mg} / \mathrm{j}$ pendant 4 jours.

Cinq jours après le début du traitement, il y a eu une très nette amélioration clinique. À six mois, il n'y a pas eu de récidive (fig. 11, 12).

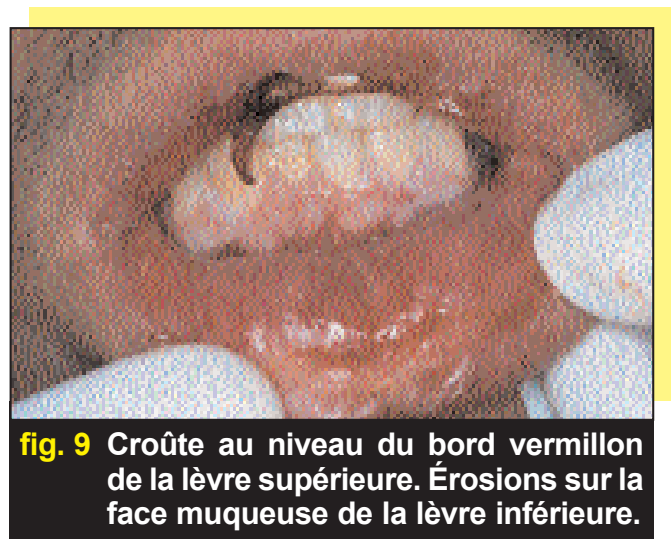



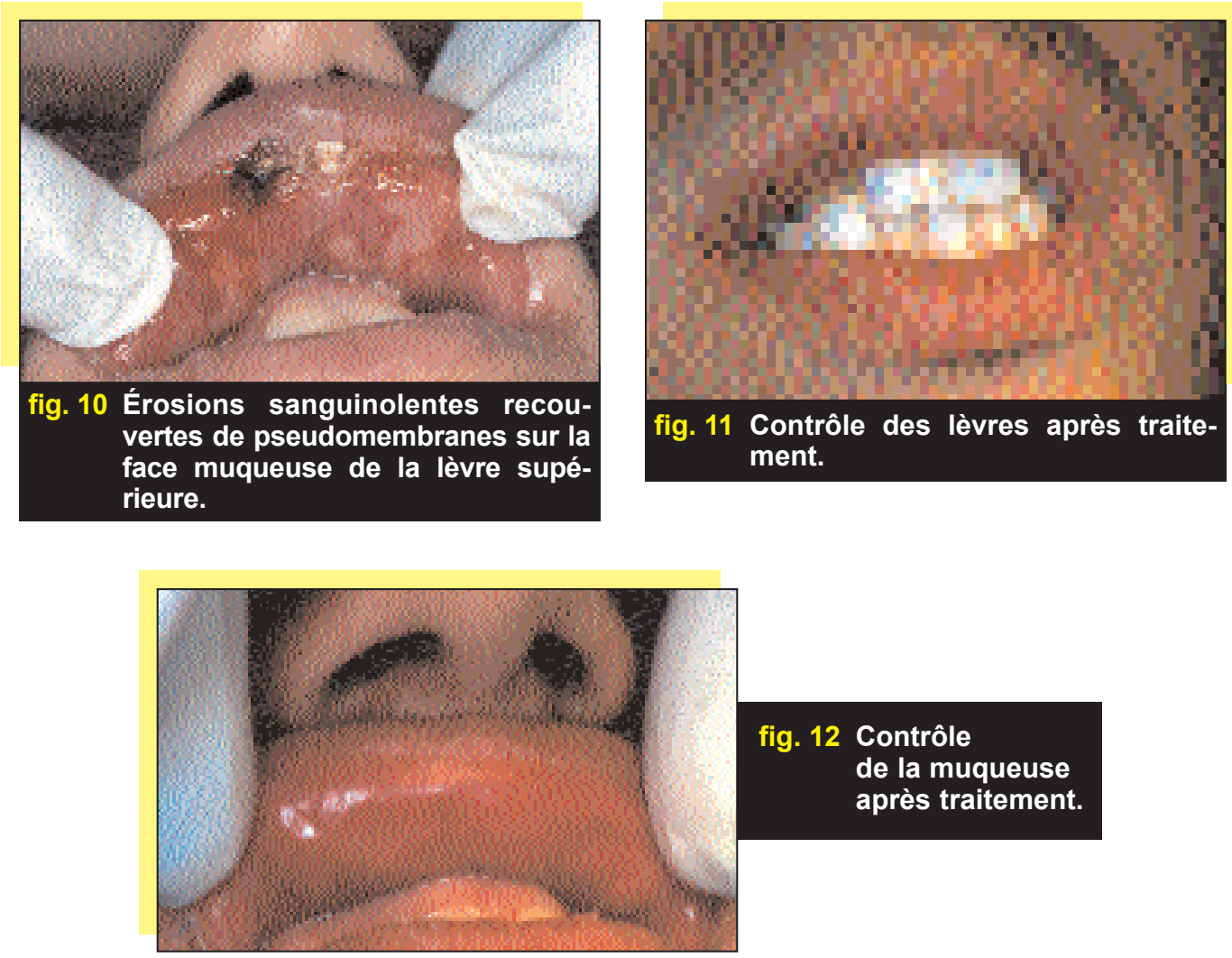

\section{cas clinique $n^{\circ} 3$ (fig. 13 à 20)}

Monsieur M., né en 1981, a été adressé en 2002 au service d'odontologie chirurgicale du CCTD pour des «aphtes multiples» et une incapacité à s'alimenter avec un malaise général. Ce patient présentait depuis 1996 des lésions buccales récidivantes chaque été.

Un bilan complet du patient a été réalisé par son médecin traitant : NFS, sérologie (HIV, hépatite B), TDM, IDR. Ce bilan était négatif.

L'examen clinique a révélé la présence de lésions à type de bulles éclatées recouvertes de dépôts fibrineux entourées d'un halo érythémateux siégeant au niveau du palais, de la langue, de la face interne des joues et des lèvres (fig. 13, 14, 15, 16). Au niveau cutané, les lésions présentaient un aspect de cocarde entourée d'un érythème (fig. 17).

$\mathrm{Au}$ niveau du bord vermillon des lèvres, les lésions étaient croûteuses et sanguinolentes.

Devant ces signes cliniques, le diagnostic de présomption a été : l'érythème polymorphe ou la pemphigoïde.

Afin de poser un diagnostic de certitude, une biopsie a été réalisée au niveau de la face interne des lèvres. 

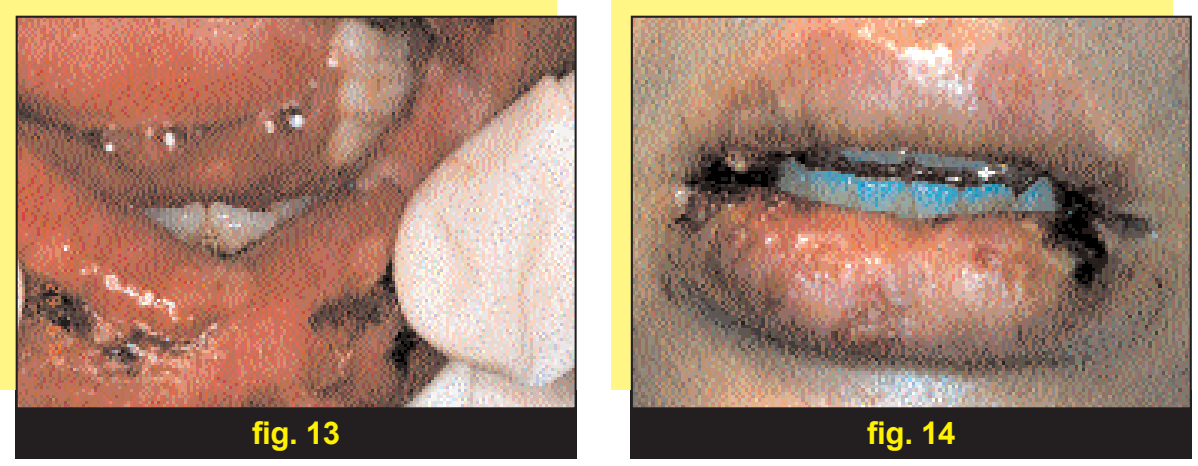

fig. 13, 14, 15 Érosions et croûtes sanguinolentes au niveau de la lèvre inférieure.
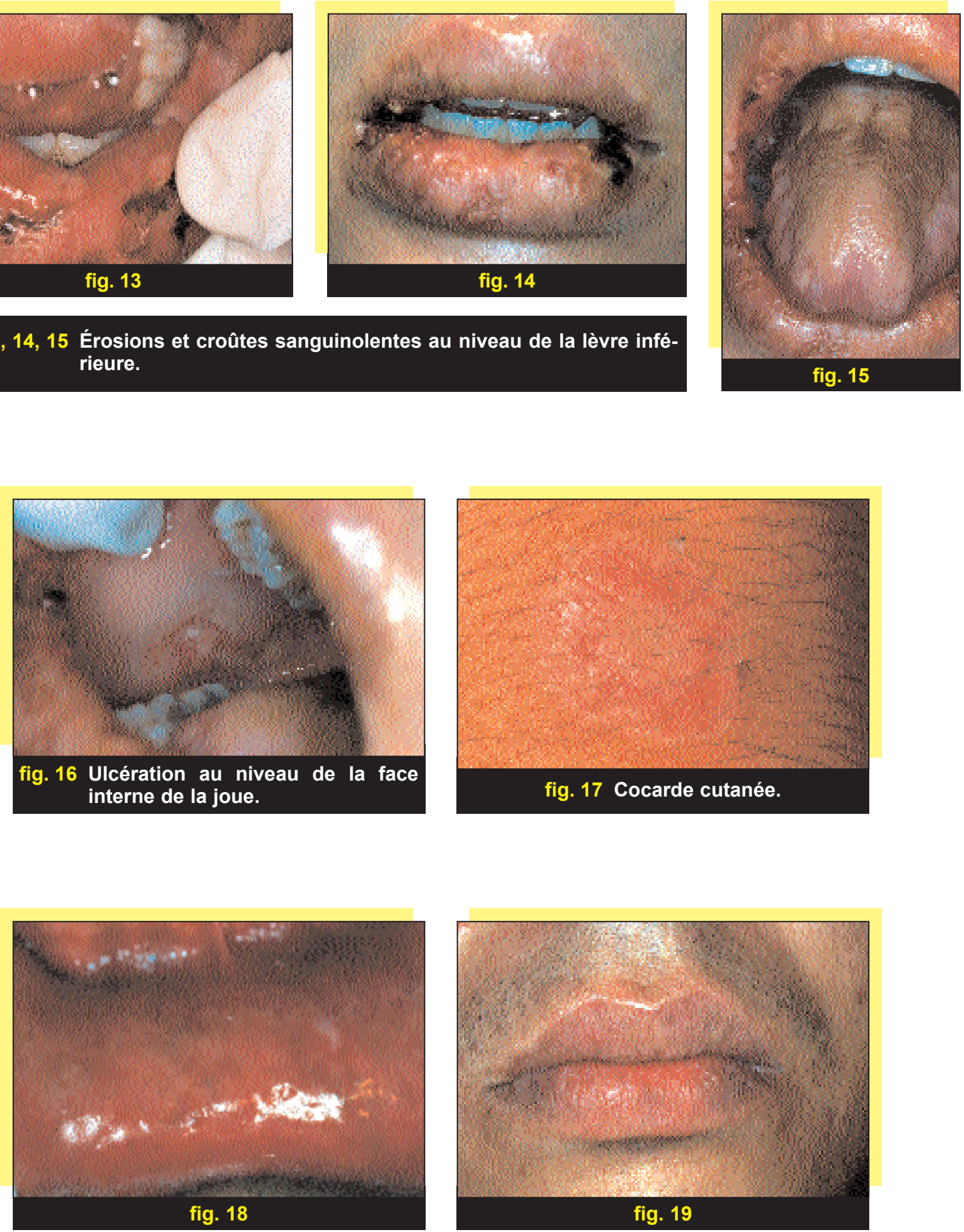

fig. 18, 19 Contrôle de la muqueuse après traitement. 


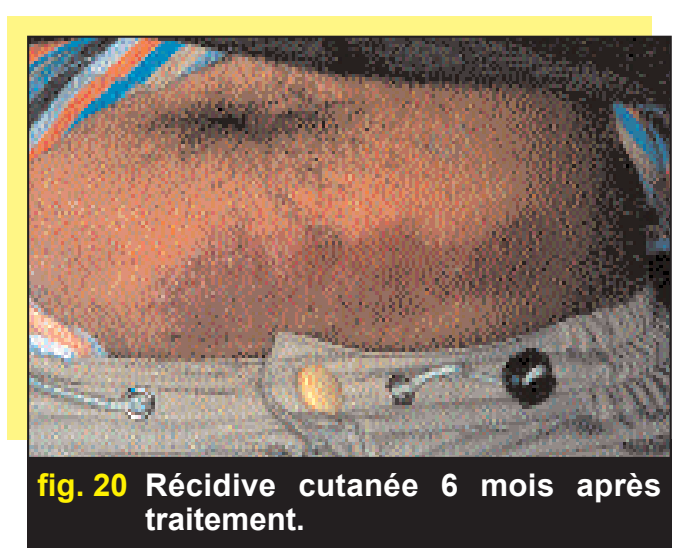

L'examen anatomopathologique a révélé un EP à localisation muqueuse buccale par la présence d'une bulle intra-épithéliale à clivage basal avec des kératinocytes nécrosés et des polynucléaires éosinophiles. Il s'agissait donc d'un EP majeur. Le traitement instauré chez ce patient a été un antiinflammatoire stéroïdien : prednisolone (Solupred $^{\circledR}$ ) $20 \mathrm{mg}, 4 \mathrm{cp} / \mathrm{j}$.

Dix jours après, il y a eu une bonne évolution (fig. 18, 19) mais six mois après, une poussée bulleuse au niveau de l'abdomen et de la nuque a été constatée sans aucune atteinte muqueuse (fig. 20).

L'étiologie exacte de l'EP n'a pu être déterminée.

\section{discussion}

L'érythème polymorphe est un syndrome éruptif aigu, récidivant, caractérisé par des lésions cutanées maculopapuleuses, bulleuses avec ou sans atteinte muqueuse.

Selon l'aspect des lésions cutanées, leur extension et le degré d'atteinte muqueuse, deux tableaux peuvent être individualisés : l'érythème polymorphe et la toxidermie bulleuse.

L'EP peut être :

- mineur : dans ce cas, la lésion élémentaire est une maculo-papule œdémateuse et érythémateuse, sans atteinte de la muqueuse[1] ;

- majeur : où il comporte des bulles et des vésicules siégeant au centre des cocardes. L'atteinte muqueuse est quasi constante. Ces lésions sont surtout buccales, oculaires et génitales. Dans sa localisation buccale, l'EP est caractérisé par une bulle ou érosion intra-buccale périodique, récurrente, ou chronique qui perturbe la phonation et la mastication. L'EP buccal survient fréquemment chez les adolescents et les jeunes adultes mais peut se produire à tout âge.

La toxidermie buccale (TB) comporte le syndrome Stevens-Johnson (SSJ) et la nécrolyse épidermique toxique (NET) que l'on distingue dans la majorité des cas par la clinique, surtout l'étendue des lésions, l'histologie des lésions cutanées et par l'étiologie[1, 2, 3, 4].

- Le syndrome Stevens-Johnson (SSJ) est caractérisé par l'importance des lésions muqueuses érosives buccales, oculaires, génitales et par des lésions cutanées peu nombreuses[1].

- La nécrolyse épidermique toxique (NET) ou syndrome de Lyell : c'est la forme grave. Les lésions muqueuses sont identiques à celles du SSJ, et les lésions cutanées peuvent atteindre 
jusqu'à 100\% de la surface corporelle[1].

Les lésions buccales peuvent être cycliques. La fréquence de ces lésions est d'environ une fois toutes les 3 semaines. La durée de chaque cycle varie de 10 jours à 6 semaines. L'étiologie est en grande partie inconnue[5].

D'un point de vue épidémiologique, l'EP est une maladie ubiquitaire, sans prédilection ethnique, qui survient à tout âge mais plus fréquemment chez l'adulte jeune. L'âge moyen est de 25 à 30 ans et un pic d'incidence dans la $3^{\mathrm{e}}$ décennie a été constaté[6]. Les sujets âgés de moins de 3 ans ou de plus de 50 ans sont rarement atteints $[7,8]$. Une prédominance masculine plus ou moins marquée est habituelle, il n'y a pas de prédominance saisonnière [9].

Concernant la physiopathologie, les différentes théories avancées font évoquer un mécanisme immuno-allergique. En effet, différents mécanismes immunitaires (complexes immuns circulants aboutissant à une libération de radicaux libres toxiques) et inflammatoires (perturbation du métabolisme de l'histamine) interviennent pour aboutir aux lésions d'EP[10].

Par ailleurs, les étiologies de l'EP sont multiples, on rapporte les infections herpétiques, les infections à mycoplasma pneumoniae et les médicaments.

\section{- infection à herpès simplex virus : (HSV)}

Dans les EP post-herpétiques, des protéines virales et des fragments d'acide désoxyribonucléique (ADN) viral sont présents dans les lésions, mais sans particule virale infectante[6]. L'HSV n'a été qu'exceptionnellement isolé à partir de culture de biopsies cutanées de lésions d'EP[6].

Plusieurs études se sont penchées sur la mise en évidence de fragments d'ADN. C'est ainsi que des techniques d'hybridation in situ ont permis de localiser les acides nucléiques viraux dans l'épiderme. En effet, la présence du fragment d'ADN correspondant à la polymérase d'HSV peut être démontrée par polymérase chain reaction (PCR) sur biopsie de lésions d'EP dans 40 à $60 \%$ des cas $[11,12,13,14]$.

La difficulté de mettre en évidence le virus ou son génome dans les lésions d'EP pourrait être due au fait que dans les lésions d'EP, seule une partie du génome est exprimée et non le génome complet de l'herpès virus simplex[10].

\section{- infections à mycoplasma pneumoniae}

Les infections à mycoplasma pneumoniae sont généralement responsables d'EP majeurs chez les enfants ou les adultes jeunes. Elles se manifestent par une toux associée à une fièvre, des myalgies et une pneumopathie atypique radiologique. Des titres élevés d'agglutinine froide et une ascension du taux d'anticorps spécifiques IgM permettent de confirmer le diagnostic $[6,15]$. Cette infection a été objectivée dans environ $5 \%$ des formes majeures d'EP[6].

\section{- les médicaments}

Les sulfamides ont été souvent impliqués dans la survenue d'un SSJ[16]. Cette fréquence élevée a été mise en évidence lors de la large utilisation des 
formes retard dans les campagnes de prévention de la méningite en 1967 au Maroc, du choléra en 1981 au Mozambique.

Les autres médicaments fréquemment incriminés sont : les antibiotiques (pénicilline), les anti-convulsivants (barbituriques, carbamazépine, Hydantoine), les antalgiques (acide acétyl salicylique), les antituberculeux, les anti-inflammatoires non stéroïdiens notamment les pyrazolés.

À côté de ces étiologies virales et médicamenteuses, on rapporte des causes diverses telles: les agents chimiques, physiques et mécaniques (bois tropicaux, tatouage, froid, soleil...), les néoplasmes et certaines maladies systémiques et enfin l'EP idiopathique ou de cause inconnue.

Parmi les trois observations rapportées, l'étiologie de l'EP n'a pu être établie avec précision que pour le cas $\mathrm{n}^{\circ} 1$ car l'interrogatoire a révélé l'absence de prise médicamenteuse et de poussées herpétiques chez les deux derniers patients.

Quant aux signes cliniques, l'EP est précédé de :

- Prodromes : un syndrome pseudogrippal est parfois noté dans la semaine précédant l'éruption.

Il semblerait être un facteur causal par action de l'agent infectieux ou par toxicité d'un médicament donné.

- Au niveau cutané, la lésion élémentaire caractéristique de l'EP est la cocarde. Cette dernière est de forme régulière, ronde, bien limitée et comprend au moins trois zones: un disque central érythémateux parfois cyanotique ou bulleux, entouré par au moins deux anneaux concentriques, l'anneau intermédiaire œdémateux, est plus pâle que le centre, et l'anneau externe est érythémateux. Cet élément a été retrouvé dans les cas 1 et 3 .

- Les lésions muqueuses sont dominées par une plaque érythémateuse associée à un œdème, sur lequel apparaît une bulle qui se perce rapidement pour laisser place à des érosions douloureuses recouvertes de pseudomembranes et entourées d'une aréole érythémateuse.

L'atteinte muqueuse est souvent synchrone de l'éruption cutanée, mais elle peut la précéder de quelques jours comme la suivre. Son intensité est indépendante de celle de l'atteinte cutanée. Les lésions buccales et péribuccales sont pratiquement constantes : bulles confluentes rapidement remplacées par de larges érosions saignantes et recouvertes d'un enduit pseudo-membraneux grisâtre.

Les lèvres tuméfiées, ulcérées et croûteuses, la face interne des joues, le vestibule, le palais et la langue, peuvent être parsemés d'érosions et d'ulcérations douloureuses. Ces différents aspects ont été retrouvés dans les trois observations. Le pourtour narinaire peut être érodé avec jetage nasal séro-sanglant. Les lésions des lèvres sont fréquentes et peuvent aboutir à une sclérose[19]. Elles sont d'une aide précieuse au diagnostic.

À côté de ces manifestations cutanéomuqueuses, l'EP peut présenter des signes systémiques dont une sensation de malaise général caractérisé par un prurit, une sensation de brûlure, de douleur, une fièvre... des myalgies, des arthralgies et des arthrites[20].

Le diagnostic positif de l'EP repose sur : 
- l'interrogatoire qui relève par un questionnaire rapide précis et orienté, le siège et la date du début de l'éruption. Les circonstances d'apparition, l'extension de l'éruption, l'existence d'événements déclenchants dans les 15 jours précédents l'éruption, notamment une prise médicamenteuse ou une lésion herpétique ;

- l'examen clinique consiste en un examen minutieux endo et exobuccal. Il permet de relever au niveau endobuccal l'emplacement, les dimensions et les caractères des lésions, la présence d'érythèmes, de vésicules, de bulles, et d'érosions.

L'examen exobuccal recherche les atteintes cutanées notamment au niveau des zones découvertes telles que mains et pieds.

- L'examen anatomopathologique :

- Le frottis : a pour but principal de réaliser le diagnostic différentiel avec le pemphigus grâce au cytodiagnostic de Tzank.

- La biopsie : elle doit être faite dans le cas où le diagnostic est incertain en particulier en l'absence des lésions en cocarde. L'aspect histologique de la biopsie de la muqueuse buccale est identique à celui de la biopsie au niveau cutané, c'est-à-dire un aspect inflammatoire ou nécrotique.

L'aspect inflammatoire est composé d'un infiltrat prédominant dans le derme superficiel. Il existe aussi des infiltrats péri-vasculaires modérés dans le derme profond, sans vascularite associée. L'exocytose est constante, proportionnelle à l'inflammation, les cellules inflammatoires sont lymphocytaires et histiocytaires. Des polynucléaires neutrophiles et éosinophiles peuvent être également présents toujours en proportions réduites[21].

La forme nécrotique est caractérisée par une dégénérescence épidermique prédominante étendue, aboutissant à un clivage sous-épidermique. L'infiltrat dermique lymphohistocytaire, modéré, prédomine dans les aires vasculaires et ne s'accompagne pas ou peu d'exocytose. Cet aspect histologique paraît plutôt être le fait du SSJ[10].

- L'examen paraclinique : consiste en un examen en immunofluorescence directe, un examen biologique et un examen radiologique.

Le diagnostic différentiel : se fait avec l'urticaire. les pathologies virales à expression muqueuse, les pathologies bulleuses telles les érosions ou ulcérations, le lichen plan bulleux, la pemphigoïde cicatricielle, la pemphigoïde bulleuse, la maladie de Ritter-Lyell, l'épidermolyse bulleuse congénitale, le syndrome de Reiter, la dermatose bulleuse à IgA linéaire, la dermatite herpétiforme et les stomatites médicamenteuses.

Le traitement de l'EP comporte trois volets, l'un symptomatique, l'autre étiologique, et enfin un traitement de fond. Le traitement symptomatique concerne les manifestations buccales et cutanéomuqueuses.

Pour ce qui est des manifestations buccales, il a pour but la prise en charge de la douleur, la prévention de la surinfection des lésions et l'amélioration de la gêne fonctionnelle et de la cicatrisation. En effet, les lésions érosives de la cavité buccale peuvent être spontanément très douloureuses et nécessiter l'instauration d'une couverture antalgique. 
Si l'utilisation de paracétamol est généralement suffisante, le recours aux dérivés morphiniques mineurs peut parfois s'avérer indispensable.

La mise en place sur les lésions d'un anesthésique topique tel que le gel de xylocaïne ou de lidocaïne, au moment des repas, permet de diminuer les sensations douloureuses lors de l'alimentation $[22,23]$.

L'infection secondaire des lésions de la muqueuse buccale est favorisée par la présence de plaque bactérienne. Elle doit être prévenue par une hygiène bucco-dentaire rigoureuse incluant l'utilisation d'antiseptiques locaux à base de chlorhexidine, de dérivés iodés ou d'hexetidine. Il est conseillé d'utiliser une brosse à dents souple et un dentifrice non irritant [19, 23].

La présence d'ulcérations plus ou moins étendues dans la cavité buccale peut entraîner des douleurs intenses lors de l'alimentation.

Les patients doivent donc éviter les aliments risquant de majorer ces douleurs, notamment les boissons gazeuses et les aliments acides ou fortement épicés et préférer une alimentation froide, voire glacée et semi-liquide.

Si la dysphagie est importante, une supplémentation nutritionnelle riche en calories peut être mise en place.

Par ailleurs, l'utilisation d'une sonde gastrique peut s'avérer nécessaire, en particulier lors d'atteintes bucco-œsophagiennes sévères[23].

Enfin, la cicatrisation des lésions buccales lorsque celles-ci sont sévères, est améliorée par l'utilisation de corticoïdes locaux, prednisolone (solupred ${ }^{\circledR}$ ) en bain de bouche ou dermocorticoïdes.
Sans corticothérapie locale, les lésions peuvent perdurer pendant 3 à 8 semaines. Les lésions labiales doivent également être protégées, soit par une crème anti-inflammatoire, soit par un simple stick labial.

Plusieurs auteurs anglo-saxons font référence à l'utilisation de pâtes adhésives à usage intrabuccal à base de carboxyméthylcellulose (Orabase ${ }^{\circledR}$ ) ou d'hydroxypropycellulose (Zilactin ${ }^{\circledR}$ ), dans le traitement des lésions ulcéroérosives de la cavité buccale.

Ces pâtes possèdent des qualités adhésives intéressantes sur la muqueuse préalablement asséchée, de l'ordre de 1 heure à 1 heure 30 pour l'Orabase ${ }^{\circledR}$ et de 3 à 4 heures pour le Zilactin ${ }^{\circledR}$.

Le Zilactin ${ }^{\circledR}$ permet en plus d'une protection mécanique de la lésion, une sédation des douleurs liée à la présence d'HPC (hydroxypropylcellulose).

L'Orabase $^{\circledR}$ est principalement utilisé pour faciliter une exposition plus longue de la muqueuse à une substance active, en général un dermocorticoïde de niveau 1 ou 2 (Clobétasol propionate à $0,05 \%$ ou Fluocinonide à 0,05\%).

Le traitement consiste en 2 ou 3 applications par jour pendant 4 semaines, après mélange en parts égales de l'Orabase ${ }^{\circledR}$ et de la substance active.

Cette thérapeutique a un effet bénéfique dans le contrôle des symptômes (érythème, douleur) et de la taille des lésions ulcéro-érosives intrabuccales.

Ces pansements intrabuccaux sont réservés à des érosions ou ulcérations de taille modérée et peu nombreuses en raison de l'inconfort qu'ils peuvent procurer aux patients[24, 25].

Par ailleurs, en ce qui concerne les manifestations cutanéo-muqueuses, le 
traitement symptomatique fait appel à un antiseptique local appliqué sur les lésions cutanéo-muqueuses bulleuses sauf en cas de décollement étendu où des pansements cicatrisants sont prescrits. Les complications locales sont surtout oculaires et nécessitent une prise en charge spécialisée rapide. Les formes graves du SSJ peuvent nécessiter une prise en charge en milieu spécialisé pour rééquilibration hydroélectrolytique, apports nutritionnels et antibiothérapie guidée par les prélèvements locaux.

Le traitement étiologique comprend :

- Aciclovir et les autres antiviraux en cas d'infection herpétique, un essai contrôlé a montré qu'un traitement continu d'aciclovir à la dose de $400 \mathrm{mg}$ deux fois par jour pendant 6 mois permettait de supprimer 60 à $80 \%$ des récurrences d'herpès et d'EP[6].

- Antibiothérapie antimycoplasme en cas d'infection par mycoplasma pneumoniae. Un effet bénéfique sur la durée de l'évolution ou l'intensité des lésions d'EP n'a jamais été prouvé.

- Il faut aussi noter qu'en cas de suspicion de toxidermie, tous les médicaments sont arrêtés.

Le traitement de fond quant à lui, comporte :

- La corticothérapie : l'intérêt éventuel des corticoïdes dans le traitement des poussées d'EP est controversé. Son utilisation est justifiée par certains du fait de l'hypothèse d'une réaction immunologique. Cependant, il n'y a jamais eu d'essai contrôlé de bonne qualité.

- La dapsone : plusieurs cas de rémission de la maladie ont été observés chez des patients traités par des doses de 100 à $150 \mathrm{mg}$ par jour. Cependant, peu d'auteurs font état de son efficacité dans le cadre de l'érythème polymorphe.

- Le thalidomide : une dose de $100 \mathrm{mg} / \mathrm{j}$ est en règle efficace initialement et l'effet se maintient avec une dose d'entretien plus faible. Cependant, compte tenu de son risque tératogène majeur, ce produit ne peut être utilisé chez les femmes en âge de procréer que dans des conditions de surveillance stricte[6].

- Le lévamisole: son efficacité a été démontrée dans le traitement de l'EP buccal et d'autres ulcérations chroniques de la cavité buccale. Il est utilisé seul ou en association avec un corticoïde dans les cas plus sévères ( 5 à 30 $\mathrm{mg}$ de prednisolone). La posologie est de $150 \mathrm{mg}$ par jour pendant 3 jours.

- L'azathioprine : plusieurs études ont démontré son efficacité dans les cas d'EP réfractaires aux traitements de première intention, en particulier les antiviraux.

Les doses quotidiennes se situent entre 100 et $150 \mathrm{mg} /$ jour.

Pour les présentes observations, la prise en charge des lésions buccales par un traitement à base d'anti-inflammatoire stéroïdien a donné à court terme d'excellents résultats.

Les récidives restent néanmoins possibles, ce qui suggère la nécessité d'un suivi régulier pluridisciplinaire, voire la mise en route d'un traitement plus efficace à base de lévamisole, ou de thalidomide.

De même, pour tous ces patients, une mise en état bucco-dentaire, bien que difficile vu les signes fonctionnels, a 
accompagné le traitement symptomatique et général.

Évolution : l'évolution naturelle de la maladie se fait par poussées successives durant une période de quelques jours à 3 semaines. La guérison définitive intervient en 2 à 4 semaines.

L'évolution du SSI est assez comparable dans le temps à celle de l'EP, mais le risque de complication est plus important.

Le délai de guérison est plus long mais en règle générale inférieur à 6 semaines. Le risque principal est la possible évolution vers un syndrome de Lyell rendant cette affection passablement létale.

\section{conclusion}

L'érythème polymorphe est une maladie relativement fréquente touchant les sujets jeunes avec une légère prédominance masculine.

On distingue l'EP mineur et majeur avec ses cocardes typiques à distribution acrale et, d'autre part, le syndrome de Stevens-Johnson et la nécrolyse épidermique toxique avec des macules et vésicules disséminées ou à prédominance centrale. L'EP, majeur ou mineur, a surtout été associé aux infections à herpès simplex virus (HVS), alors que le syndrome de Stevens-Johnson est principalement dû à des réactions médicamenteuses. Quant à l'EP buccal, c'est une entité moins bien reconnue comme le reste $d u$ spectre d'EP.

L'étiologie de l'EP est obscure. Le diagnostic est clinique: syndrome cutanéo-muqueux aigu caractérisé par des lésions cutanées en cocarde très typiques siégeant symétriquement sur les faces d'extension des membres. Les lésions muqueuses érosives, inconstantes, font la sévérité du tableau.

Le traitement est symptomatique : antisepsie des lésions bulleuses. La corticothérapie générale n'est pas toujours bénéfique dans le traitement d'un érythème polymorphe installé. Elle peut se discuter pour la prévention des formes récidivantes. L'aciclovir peut avoir une place dans les récurrences post-herpétiques.

L'évolution, spontanément régressive, est le plus souvent bénigne.

Le rôle $d u$ chirurgien-dentiste est fondamental dans le diagnostic précoce de l'EP, la surveillance clinique et la participation au traitement, tout en collaborant avec le dermatologue et l'ophtalmologue.

\section{bibliographie}

1. Touraine R Abrégé de dermatologie clinique et vénérologie. Paris : Masson, 1991.
2. Assier $\mathrm{H}$, Bastuji-garin $\mathrm{S}$, Revuz J, Rougeau JC. Erythema multiforme with mucous membrane involvement and Stevens-Johnson syndrome are clinically different disorders with 
distinct causes.

Arch Dermatol 1995;131: 539-543.

3. Auquier-Dunant $A$, Mockenhaupt M, Naldi L, Correia O, Schroder W, Rougeau JC.

Correlations between clinical patterns and causes of erythema multiforme majus, Stevens-Johnson syndrome and toxic epidermal necrolysis. Arch Dermatol 2002;198: 1019-1024.

4. Bastuji-Garin S, Rzany B, Stern RS, Shear NH, Naldi L, Roujeau JC. Clinical classification of cases of toxic epidermal necrolysis, Stevens-Johnson syndrome, and erythema multiforme. Arch Dermatol 1993:92-96.

5. Ayango L, Rogers RS. Oral manifestations of erythema multiforme. Dermatol Clin 2003;21(1): 195-205.

6. Tesici A, Rougeau JC. Érythème polymorphe. Paris : Elsevier, Encycl Med Chir Dermatologie 2001; 98-265-A-10, 7p.

7. Johnson GA, Ghura HS, Carter E,

Graham-brown RAC. Neonatal erythema multiforme major. Clin Exp Dermatol 2002; 27(8):661-664.

8. Nanda $S$, Pandhi D, Reddey BSN. Erythema multiforme in a 9 day old child. Pediatr Dermatol 2003; 20(5):454-455.

9. Martin-Mateos MA, Roldan ROS A, Munoz-Lopez F.
Erythema multiforme: a review of twenty cases. Allergol Immunopathol 1998;26(6):283-287.

10. Barbaud A.

Physiopathologie de l'érythème polymorphe. Ann Dermatol Venereol 1998;125:799-805.

11. Aslanzadeh J, Helm KF, Espy MJ, Muller SA, Smith TF.

Detection of HSV specific DNA in biopsy tissue of patients with erythema multiforme by polymerase chain reaction.

Br J Dermatol 1992;126: 19-23.

12. Aurelian L, Ono F, Burnett $J$.

Herpes simplex virus (HSV) associated erythema multiforme (HAEM):

A viral disease with an autoimmune component.

Dermatology Online Journal 2003;9(1):1.

13. Kamauh MD, Zainal $D$, Mokhtar N, Nazmi N. Erythema multiforme, Stevens-Johnson syndrome and toxic epidermal necrolysis in northeasterm Malaysia. Int J Dermatol 1998;37: 520-523.

14. Sun $Y$, Chan $R$, Tan $S$, Peilinng P. Detection and genotyping of human herpes simplex virus in cutaneous lesions of erythema multiforme by Nested PCR. J Med Virol 2003;71(3):423-428.

15. Reichert-Penetret $S$, Barbaud A, Antunes A, Borsa-Dorion A,
Vidailhet M, Schmutz JL. An unusual form of Stevens-Johnson syndrome with subcorneal pustules associated with mycoplasma pneumoniae infection.

Pediatr Dermatol 2000; 17(3):202-204

16. Chakroun $\mathrm{M}$, et al. Syndrome de Stevens-Johnson au cours d'une toxoplasmose oculaire traitée par la sulfadiazine. Nouv Dermatol 1997; 16:326-328.

17. Piette E, Reychler H. Traité de pathologie buccale et maxillo-faciale. Bruxelles :

De Boeck Université, 1991:281-282.

18. Calzavara Pinton PG, Venturini M, Capezzera R, Zane C, Fachetti F. Photosensitive erythema multiforme and erythema multiforme like polymorphous light eruption.

Photodermatol

Photoimmunol Photomed 2003;19:157-159.

19. Mareira Marinho LH, Haj M, Malaco Pereira LF, Horizonte B, Gerais M. Lip adhesion: An unsual complication of erythema multiforme. Oral Surg Oral Med Oral Pathol

Oral Radiol Endod 1999;88(2):167-169.

20. Molnar I, Matulis M. Arthritis associated with recurrent erythema multiforme responding to oral acyclovir. Clin Rheumatol 2002;21(5): 415-417. 
21. Cote B, Wechsler J, Bastuji-Garin S, Assier H, Revuz J, Rougeau JC. Clinicopathologic correlation in erythema multiforme and Stevens-Johnson syndrome.

Arch Dermatol 1995;131: 1268-1272.

22. Provost $T$, Weston $W$, Huff J.

Bullous diseases.

Saint-Louis : Mosby, 1993:279.
23. Vaillant $L$, Goga D. Dermatologie buccale. Paris : Doin, 1997.

24. Lozada Nur F, Huang MZ, Zhou G.

Open preliminary clinical trial of chobetasol propionate ointment in adhesive paste for treatment of chronic oral vasculoerosive diseases. Oral Surg Oral Med Oral Pathol 1992;71: 283-287.
25. Lozada Nur F, Miranda C, Maliksi R.

Double blind clinical trial of $0,05 \%$ clobetasol propionate ointment in Orabase and $0,05 \%$ fluocinonide ointment in Orabase in the treatment of patients with oral vasculoerosive diseases.

Oral Surg Oral Med

Oral Pathol 1994;77: 598-604.

\section{SUMMARY}

\section{The multiform erythema: about three cases}

I. BENYAHYA, L. KISSI, K. GUESSOUS

The erythema multiforme (EM) is an acute eruptive syndrome. It can occur with or without mucous attack. Its elementary lesion at the mucous level is a bubble which quickly bursts and leaves more or less surface and painful erosions. At the cutaneous level, the lesion in rosette sits primarily on the level of the ends. The positive diagnosis is clinical and anatomopathologic. The treatment of the EM comprises three shutters, one symptomatic, the other ethiologic, and finally a basic treatement which calls upon corticoids, but also with the levamisole, dapsone, thalidomide, etc. The evolution although generally benign can be enamelled periods of repetition. The role of the dental surgeon supported through three observations is fundamental in the diagnosis and the assumption of responsibility therapeutic in collaboration with the dermatologist and the ophtalmologist.

keywords: erythema multiforme, Stevens-Johnson syndrome, cockade, stomatitis. 